\title{
Microbiological Profile and Antimicrobial Resistant Pattern of Blood Culture Isolates, Among Septicaemia Suspected Patients
}

\section{ABSTRACT}

Introduction: Microorganisms cause septicaemia,a systemic disease due to their multiplication and toxins in the blood. These bloodstream infections constitutes significant public health problems and are a major cause of morbidity and mortality in the hospitalised patients and require rapid antimicrobial treatment. Infection by MDR organisms are more likely to increase the risk of death in these patients.

Aim: To determine the microbiological profile and antimicrobial resistant patterns among patients suspected to have septicaemia at a tertiary care hospital.

Materials and Methods: A total number of 121 patients were included in this study suspected to have septicaemia. The blood culture samples were processed and identified in the microbiology laboratory according to CLSI guidelines in RMCH\&RC over a period of six months. Drug resistant strains in primary screening were further processed for ESBL and MRSA by standard guidelines.

Results: Out of 121 patients, 27(22.3\%) developed septicaemia with the positive blood culture. Of the 27 positive culture, $24(88.9 \%)$ showed bacterial growth, in which $16(59.3 \%)$ were gram negative bacilli, $8(29.6 \%)$ were gram positive cocci and $3(11.1 \%)$ isolates were gram positive budding yeast cells. Most common isolates were
E.coli (22\%), Klebsiella pneumoniae (22\%), Coagulase negative Staphylococcus (CONS) spp. (15\%) followed by Pseudomonas aeruginosa (11\%) S.aureus (11\%), Klebsiella oxytoca (4\%), Enterococcus faecalis(4\%) and Candida spp (7\% C.glabrata and 4\% C.krusei) was mainly isolated in neonates. Maximum isolated strains showed high resistance towards penicillins, cephalosporins and fluoroquinolones. S. aureus and CONS were mostly resistant to co-trimoxazole and erythromycin. Resistance to imipenem was seen approximately in $66 \%$ in E.coli, Klebsiella pneumoniae and Pseudomonas spp. ESBL producers among the E.coli and Klebsiella isolates were $50 \%$ and $66.6 \%$ respectively, while $33.3 \%$ were MRSA and 50\% MR-CONS among Gram positive cocci. Tigecycline and polymyxin-B were the most effective antimicrobial agents for Gram-negative bacteria, while linezolid, teicoplanin for Gram-positive bacteria.

Conclusion: The present study provides much needed information on the prevalence of bacterial pathogens in blood stream infections and also demonstrates the presence of fungaemia due to non-Candida albicans spp., which highlights the need for periodic surveillance of etiologic agents, their antibacterial and antifungal susceptibility pattern. Also changing trends in the distribution of Candida species to prevent further emergence and spread of resistant pathogens.

Keywords: Blood stream infections, Drug resistance

\section{INTRODUCTION}

Blood stream infections cause significant morbidity and mortality worldwide and among the most common health care associated infection [1]. Bacteraemia and fungaemia are one of the cause for high mortality rate (20\%-50\%) [2]. Septicaemia may be a transient, self limited phenomenon without clinical consequences, it frequently reflects the presence of serious infection [3]. It has been confirmed by culture that is associated with clinical manifestation and systemic response [4-6]. It is often associated with hospitalization, insertion of foreign bodies such as catheters into blood vessels, and other predisposing factors like ICU, lapses in handwashing, and non-adherence to infection control practices of medical staff. Genitourinary tract, intraabdominal foci and respiratory tract are the common sources of blood stream infections [2,4]. Organism isolated from blood culture vary according to geographical distribution and development of multidrug resistant organism is of great concern as they prolong hospital stay. Increase cost of treatment and can be a cause of high mortality [7]. Blood 
culture is the gold standard for the diagnosis [6]. The changes in the local patterns of infection and susceptibility to various antibiotic should be critically evaluated periodically [3]. Therefore, this study was aimed at finding the resistance pattern of the isolates from blood in a tertiary care centre, which can help the clinician to provide empirical treatment.

\section{MATERIALS AND METHODS}

This was a prospective study conducted in the Rama Medical College Hospital and Research Centre, Kanpur in the Department of Microbiology over a period of six months from January 2015 to June 2015. A total of 121 blood culture samples were analysed. Blood samples were collected under aseptic precautions using a sterile syringe after applying tourniquet. From an adult $10 \mathrm{ml}$, children $5 \mathrm{ml}$, and neonates $1 \mathrm{ml}$ of blood into BHI Broth (Hi Media, Mumbai, India),70 $\mathrm{ml}$ and $20 \mathrm{ml}$ bottles, from different wards and critical care units from septicaemia suspected patients was collected. All blood cultures were processed in laboratory using standard procedure by conventional method. After $24 \mathrm{hrs}$ blood culture samples were sub-cultured onto Blood agar and Mac-Conkey agar to look for growth. From the obtained growth isolated colonies were used for gram's staining and biochemical tests for the differentiation of organism and antibiotic sensitivity was done by Kirby Bauer's method according to CLSI guidelines [8], and no growth plates were incubated for further 24 hours. Subcultures from blood culture bottles were done on $2^{\text {nd }}, 4^{\text {th }}$ and $6^{\text {th }}$ day, Samples were reported as no growth after 7 days of aerobic incubation. Septicaemia suspected patients who were not given antibiotics were included in the study, and patients already on antibiotics before the blood culture were excluded from the study. The antibiotics which were tested were ampicillin $(10 \mathrm{mcg})$, piperacillin/tazobactam (100/10mcg), ofloxacin (10 mcg), oxacillin (1 $\mathrm{mcg})$, cefotaxime $(30 \mathrm{mcg})$, ceftazidime $(30 \mathrm{mcg})$, cefepime (30mcg), cefoperazone/sulbactum (75/30mcg), cefoxitin (30mcg), amikacin (10mcg), gentamicin (10mcg), ciprofloxacin (5mcg), co-trimoxazole(1.25/23.75mcg), tetracycline (30mcg), polymyxin B (300mcg), colistin (10mcg), tigecycline $(15 \mathrm{mcg})$, erythromycin $(15 \mathrm{mcg})$, vancomycin (30mcg), linezolid (10mcg), teicoplanin (30 mcg). Drugs used were from Hi-Media (Mumbai, India). The screened strains were further processed for ESBL and MRSA according to CLSI guidelines [8]. Ethical clearance was obtained from the institute.

\section{RESULTS}

Out of 121 blood cultures, 27(22.3\%) gave positive result [Table/Fig-1]. Bacteraemia was seen in 24 patients (88.9\%), and fungaemia was seen in three cases (11.1\%). The majority of septicaemia patients were adults (>18 yrs) (37.0\%) and neonates (25.9\%) [Table/Fig-2]. In positive isolates males were 16(59\%) and females were 11(41\%). [Table/Fig-3] Among positive blood culture isolates Gram-negative and Grampositive bacteria constituted $59.3 \%$ and $29.6 \%$, respectively and $11.1 \%$ were Candida. The most common pathogens found was E.coli (22\%), Klebsiella pneumonia (22\%) followed by Coagulase negative Staphylococcus (CONS) spp.(15\%), Pseudomonas aeruginosa (11\%), S.aureus (11\%), Klebsiella oxytoca (4\%), Enterococcus foecalis (4\%) and Candida spp. was mainly isolated in neonates (7\% C. glabrata and $4 \%$ C.

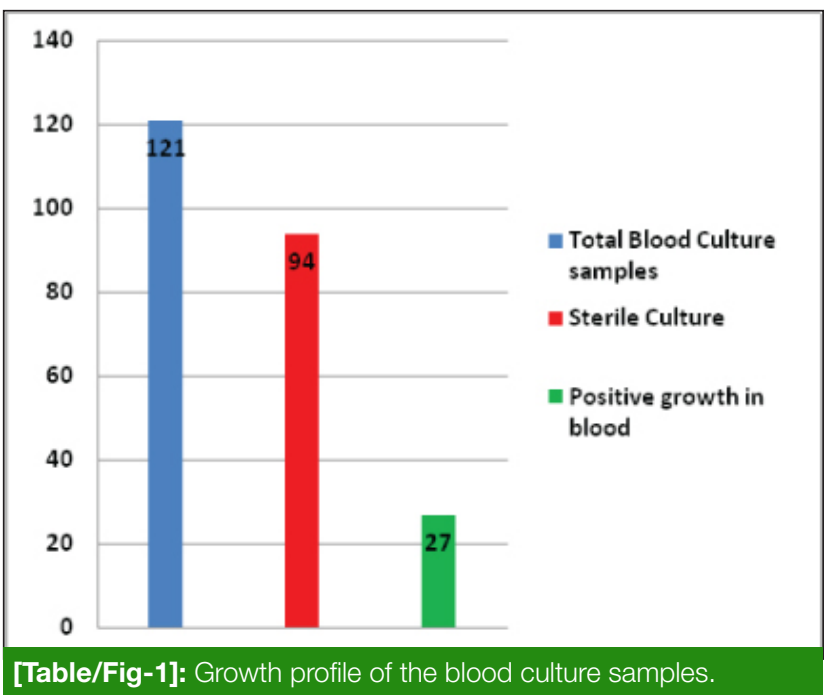

\begin{tabular}{|l|c|c|c|}
\hline Age distribution & Growth & Sterile & Total \\
\hline Infant $(<1 \mathrm{yr})$ & $7(25.9 \%)$ & 30 & 37 \\
\hline Children (1-12) & $6(22.2 \%)$ & 15 & 21 \\
\hline Adolescent (13-18) & $4(14.8 \%)$ & 23 & 27 \\
\hline Adult $(>18)$ & $10(37.0 \%)$ & 26 & 36 \\
\hline Total & 27 & 94 & 121 \\
\hline
\end{tabular}

[Table/Fig-2]: Age distribution of the blood culture samples.
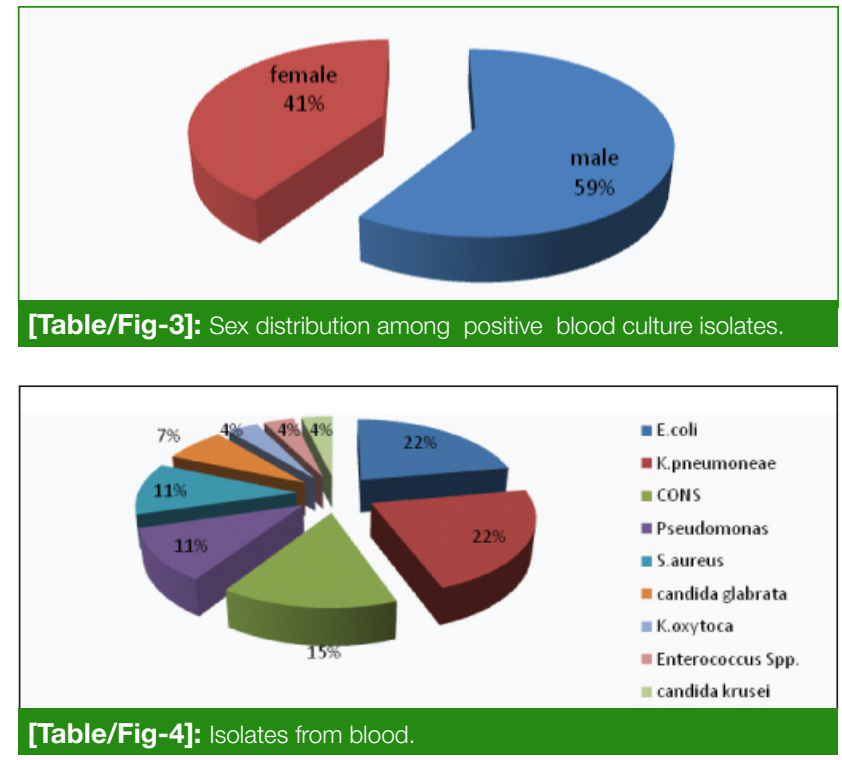

National Journal of Laboratory Medicine. 2016 Jan, Vol 5(1): 17-21 
krusei) [Table/Fig-4]. Antibiotic sensitivity pattern of different isolates were showed in [Table/Fig-5-8].

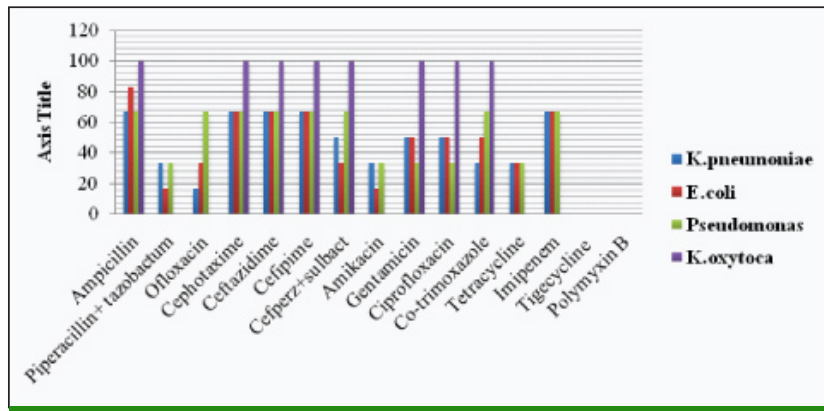

[Table/Fig-5]: Antibiotic resistance pattern of Gram negative bacilli.

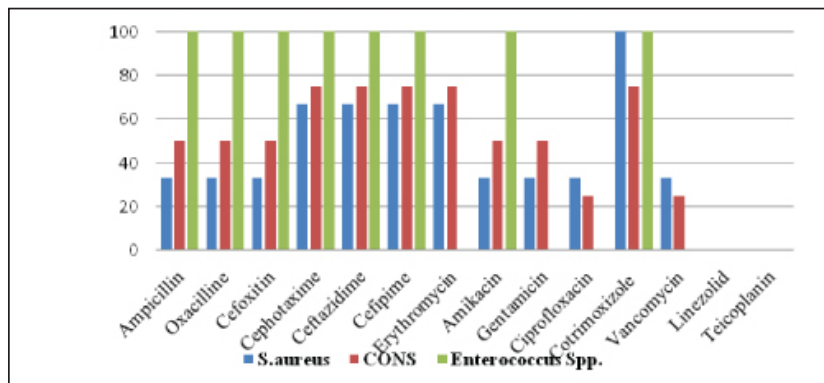

[Table/Fig-6]: Antibiotic resistance pattern of Gram positive cocci.
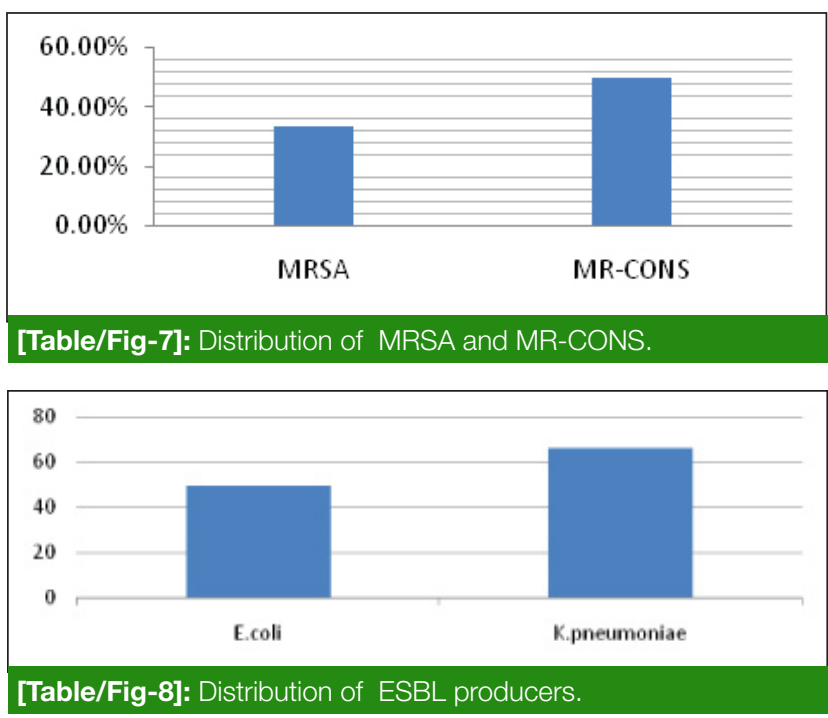

\section{DISCUSSION}

In our study a high prevalence rate of $27(22.3 \%)$ was seen. Gram negative organism was the predominantly seen. Isolates were resistant to most commonly used antibiotics. Multidrug resistant bacterial strains are emerging as a major problem in the management of sepsis. The amount and number of blood culture samples included in the studies could be one of the reasons for variation in the prevalence rate [9]. The prevalence rate of $22.3 \%$ in the present study, which is approximately similar to Arora et al., [10] 20.2\% and lower than the studies done in India, by Khanal et al.,[11] and Sharma et al., [12], have reported high frequency of positive blood cultures accounting for 44\% and 33.9\% respectively. While other studies showed low prevalence by Mehdinejad M et al., [13] Vanitha et al., [14] and Mehta et al., [15] 5.6\%, 8.39 and 9.94\% and the variation may be due to the different methodology used and the area of study, because of regional variation known to occur.

Similar, to present study in most of the studies, gramnegative bacilli have taken over the gram-positive organisms, especially in hospital settings. In our study E.coli, Klebsiella, pseudomonas was most predominant gram negative isolates. Other authors also found similar findings with addition of Acinetobacter spp, Enterobacter spp and Salmonella spp $[12,14]$.

Among gram positive pathogen CoNS and S.aureus were most common isolates similar to Vanitha et al., [14] and Rao et al., [16] though CoNS when isolated from blood cultutres are often considered as skin contaminants [7], but repeated isolation of CoNS suggests that infections by these agents constitute a significant threat.

In other studies the incidence of Candidemia was much higher (20.29\%) [16] mainly due to C.albicans while in present study $11 \%$ of positive blood culture gave growth of non-albicans candida spp (7\% C.glabrata and 4\% C. krusei) similar to other studies $[14,17]$. Candidemia in our study was due to non-albicans species, and all the cases were seen in neonates. This could probably be due to increased use of invasive devices, and broad spectrum antibiotics in NICU and PICU [18]. Prevalence of neonatal septicaemia in our study was $25.9 \%$ which was comparatively lower than other studies which showed a rate of $56 \%$ and $36 \%[12,19,20]$, the reason for this could be the less number of sample included in the studies, or anaerobic bacteraemia or a false history from patients in use of antibiotics. High prevalence of septicaemia in other studies may be due to low socioeconomic status of their parents, poor hygiene practices, bottle feeding and high incidence of delivery at home [21].

Septicaemia was caused by only one organism in our study similar to other studies [22]. Septicaemia of polymicrobial etiology observed in other studies [23-25] is significant. Some microbiologist considers polymicrobial growth as a contamination, but sepsis should be clinically correlated [26]. The clinician should be caution, when septicaemia is due to the multiple microbes not neglecting it as contaminants, but consider it as a pathogen if it is suspected to be isolated from the hospital environment [21]. In our study males were slightly more affected than female but there is no effect of gender in blood culture positivity rate.

In our study $>60 \%$ of strains were resistant to ampicillin among the gram positive cocci similar to other studies 
$[27,28]$. Resistance to cephalosporins was also observed with Staphylococcus aureus and Enterococcus faecalis in the present study. Among all Gram positive cocci 33.3\% were MRSA and 50\% were MR-CONS. This could be due to the indiscriminate use of these drugs especially third generation cephalosporins in hospitals as an emergency empirical therapy, as reported by Nathisuwan et al., [29] and Vanitha $R$ et al., [14] The study also showed that Staphyloccocus aureus was found to be mostly sensitive to vancomycin, Teicoplanin, linezolid and ciprofloxacin as reported by other authors also [14].

One study of Asrat D et al., [30] found only gentamicin and kanamycin were relatively effective against gram negatives and in our study approx $50 \%$ of strains were sensitive to gentamicin. Piperacillin + tazobactum, amikacin, tigecycline, polymyxin B were most effective drug. Ofloxacin were sensitive to Klebsiella species and E.coli but more than $60 \%$ Pseudomonas was resistance. In the present study $50 \%$ of E.coli and $66.6 \%$ of Klebsiella were ESBL producer which is higher than Kalpeshet al., [7], Kavitha et al., [31], and Arora et al., [10] who reported ESBL producers as 39.6\%, 32\% and $34.4 \%$ respectively found only gentamicin and kanamycin were relatively effective against gram negatives and in our study approx $50 \%$ of strains were sensitive to gentamicin.

\section{LIMITATION}

The limitations of this study is that though the fungaemia was seen due to Candida isolates, antifungal susceptilibility was not done due to cost constraints.

\section{CONCLUSION}

The present study provides much needed information on the prevalence of bacterial pathogens in blood stream infections predominantly gram negative bacilli were isolated from blood and non-albicans Candida spp. was mainly isolated in neonates. This study highlights the increase in antimicrobial resistant bacteria that may be due to poor infection control practices and inappropriate use of antibiotics. The key to control of antibiotic resistant pathogens is to strictly adhere to infection control practices and mandates antibiotic policy on a large scale for every hospital.

\section{REFERENCES}

[1] Negussie A, Mulugeta G, Bedru A et al. Bacteriological profile and antimicrobial sensitivity pattern of blood culture isolates among septicemia-suspected children at Tikur Anbessa Specialized Hospital and Yekatit 12 Hospital, Addis Ababa, Ethiopia. Crit Care. 2013; 17(Suppl 4): P11.

[2] Diekma DJ, Beekman SE, Chapin KC. Epidemiology and outcome of nosocomial and community on set blood stream infection. J Clin Microbiol. 2003;41:3655-60.

[3] Connie R. Mahon. Textbook of Diagnostic Microbiology. In $5^{\text {th }}$ edition. Bacteremia and Sepsis. Updated by Paula Mister, Donald C. Lehman.pg- 868-83.
[4] Wynn JL, Seed PC, Cotten CM. Does IV IG administration yield improved immune function in very premature neonates? $\mathrm{J}$ Perinatol. 2010;17:635-42.

[5] Sharma M, Yadav A, Yadav S, Goel N, Chaudhary U. Microbial profile of septicemia in children. Indian J Practicing Doctor. 2008; 17:9-10.

[6] Prabhu K, Bhat S, Rao S. Bacteriologic profile and antibiogram of blood culture isolates in a pediatric care unit. J Lab Phys. 2010;17:85-88.

[7] Gohel K, Jojera A, Soni S et al. Bacteriological profile and drug resistance patterns of blood culture isolates in a teritiary care nephrourology teaching institute. Biomed Res Int. vol. 2014, Article ID 153747, 5 pages, 2014. doi:10.1155/2014/153747.

[8] CLSI Performance standard for Antimicrobial susptibility testing; twenty fourth informational supplement CLSI document M100S24. Wayne, PA clinical Laboratory Standard Institute; 2014.

[9] Lee A, Mirrett S, Reller LB, Weinstein MB. Detection of blood stream infections in adults: How many blood cultures are needed? J Clin Microbiol. 2007; 45:3546-48.

[10] Arora U. Devi P. Bacterial profile of blood stream infections and antibiotic resistance pattern of isolates. J K Sci. 2007; 9:18690.

[11] Khanal B. Harish BN, Sethuraman KR, Srinivasan S. Infective endocarditis: Report of prospective study in an Indian Hospital. Trop Doct. 2002; 32:83-85

[12] Sharma PP, Halder D, Dutta AK. Bacteriological profile of neonatal septicemia. Ind Pediatr. 1987;24:1011-17.

[13] Mehdinejad M, Khosravi AD, Morvaridi A. Study of prevalence and antimicrobial susceptibility pattern of bacteria isolated from blood cultures. Journal of Biological Sciences. 2009; 9: 24953.

[14] Vanitha RN, Kannan G, Venkata N ,Vishwakanth D, Nagesh V, Yogitha $\mathrm{M}$ et al. A Retrospective study on blood stream infections and antibiotic susceptibility patterns in a tertiary care teaching hospital. International Journal of Pharmacy and Pharmaceutical Sciences. 2012;4(1): 543-48

[15] Mehta M , Dutta P, Gupta V. Antimicrobial susceptibility pattern of blood isolates from a teaching hospital in North India. Jpn J Infect Dis. 2005;58:174-76.

[16] Rao MSS, Surendernath M, Sandeepthi M. Prevalence of neonatal candidemia in a tertiary care institution in Hyderabad, South India. Int J Res Med Sci. 2014; 2(3): 1016-19.

[17] Narain S, Shastri JS, Mathur M, Mehta PR. Neonatal systemic candiasis in a tertiary care center. India Journal of Medical Microbiology. 2003; 21:56-58.

[18] Jaswinder KO, Chand W, Neeraj G, Reena R, Datta S, Kamaljeet P. Non-albicans Candida species in blood stream infections in a tertiary care hospital at New Delhi, India. Indian J Med Res. 2012;136: 997-1003

[19] Tsering DC et al. Bacteriological profile of septicaemia and the risk factors in neonates and infants in Sikkim. J. Glob infect Dis. 2011;3(1):42-45

[20] Mondal GP, Raghavan M et al. Neonatal septicaemia among inborn and out born babies in a referral hospital. Indian $\mathrm{J}$ Pediatr. 1991:58:529-33.

[21] Komolafe AO, Adegoke AA. Incidence of bacterial Septicaemia in Ile-Ife Metropolis, Nigeria. Malaysian J Microbio. 2008;4(Suppl 2):51-61. 33

[22] Angyo IA, Opkeh ES, Opajobi SO. Predominant bacterial agents of childhood septicaemia in Jos. Niger J Med. 2001; 10:75-77.

[23] Ghanshyam DK, Ramachandram VC, Piyush G. Bacteriological analysis of blood culture. Malaysian J Microbio. 2008;4(Suppl 2):51-61. 30 
[24] Obi CL, Mazarura E. Aerobic bacteria isolated from blood cultures of patients and their antibiotic susceptibilities in Harare, Zimbabwe. Cent Afr J Med. 1996, 42(Suppl 12):332-36.

[25] Ghanshyam DK, Ramachandram VC, Piyush G. Bacteriological analysis of blood culture isolates from neonates in a tertiary care hospital in India. J Health Popul Nutr. 2002, 20(Suppl 4):343-47.

[26] Dagnew M, Yismaw G et al. Bacterial profile and antimicrobial susceptibility pattern in septicemia suspected patients attending Gondar University Hospital, Northwest Ethiopia. BMC Res Notes. 2013; 6: 283.

[27] Guha DK, Jaspal D, Das KMS, Guha RA, Khatri RL, Srikumar R. Outcome of neonatal septicemia: A clinical and bacteriological profile. Indian Pediatr. 1978;15:423-27.
[28] Karki S, Rai GK, Manandhar R. Bacteriological analysis and antibiotic sensitivity pattern of blood culture isolates in Kanti Children hospital. J Nepal Paediatric Soc. 2010; 30(2):94-97.

[29] Nathisuwan S, Burgess DS, Lewis II JS. Extended spectrum lactamases: Epidemiology, detectionand treatment. Pharmacotherapy. 2001;21:920-28.

[30] Asrat D, Amanuel YW. Prevalence and antibiotic susceptibility pattern of bacterial isolates from blood culture in Tikur Anbassa Hospital, Addis Ababa, Ethiopia. Ethiop Med J. 2001;39(2):97104.

[31] Prabhu K, Bhat S, and Rao S. Bacteriological profile and antibiogram of blood culture isolates in a pediatric care unit. Journal of Laboratory Physicians. 2010;2:85-88.

\section{AUTHOR(S):}

1. Dr. Nidhi Pal

2. Dr. Ramamurthy Sujatha

\section{PARTICULARS OF CONTRIBUTORS:}

1. Tutor, Department of Microbiology, Rama Medical College Hospital \& Research Centre, Mandana, Kanpur, U.P, India.

2. Professor and HOD, Department of Microbiology, Rama Medical College Hospital \& Research Centre, Mandana, Kanpur, U.P, India.

\section{NAME, ADDRESS, E-MAIL ID OF THE CORRESPONDING AUTHOR:}

Dr. Ramamurthy Sujatha,

Professor and HOD, Department of Microbiology, Rama Medical College Hospital \& Research Centre, Mandana, Kanpur, U.P, India.

E-mail: drsujatha152@gmail.com

FINANCIAL OR OTHER COMPETING INTERESTS: None.

Date of Publishing: Jan 01, 2016 